\title{
Neuropsychological profiles in children and young adults with spina bifida
}

\author{
C. Rendeli ${ }^{1}$ (D) E. Ausili ${ }^{1}$ - R. Moroni ${ }^{2}$ (D) $\cdot$ M. Capriati $^{1} \cdot$ L. Massimi $^{3} \cdot$ C. Zanetti $^{1}$
}

Received: 14 December 2020 / Accepted: 9 February 2021 / Published online: 11 March 2021

(C) The Author(s) 2021

\begin{abstract}
Purpose A total of 43 Italian children, aged between 6 and 16 years, diagnosed with spina bifida, myelomeningocele, and shunted hydrocephalus have been described clinically and completed a neuropsychological battery in order to evaluate their cognitive, personality, and behavior profile.

Methods Enrolled children underwent cognitive assessment by means of the Weschler WISC-IV cognitive test and assessment of the attention sustained through the LEITER test. In addition, parents were asked, in order to obtain a personality and behavior profile of the children, to fill in a "CBCL 6-18 years" questionnaire and to fill in a Barthel Index questionnaire.

Results Processing Speed Index of the WISC-IV QI scale was statistically significant $(p=0.027)$, with the highest value presented by autonomous patients $(95.8 \pm 12.8)$ and the lowest by patients using a wheelchair $(75.5 \pm 19)$. WISC-IV QI mean value is $98( \pm 15.7)$ for lipoma patients and $78.7( \pm 17.6)$ for LMMC and MMC patients $(p=0.001)$. In more detail, Perceptual Reasoning $(p<0.0005)$, Working Memory $(p=0.01)$, and Processing Speed Index $(p=0.001)$ highlighted a significant difference between the groups. The attention sustained subscale of the LEITER presented a mean of $6.9( \pm 3.1)$ for lipoma patients and a men value of $4.6( \pm 3.1)$ for LMMC and MMC patients $(p=0.024)$. Patients with hydrocephalus had statistically significant worse cognition and autonomy (Barthel Index) score $(p<0.001)$ compared with those without hydrocephalus, and normal scores regarding attention and depression scales.

Conclusion These results can be useful in planning dedicated therapeutic protocols such as suitable rehabilitation treatments, speech therapy, psychomotor skills, and cognitive enhancement and to develop prevention protocols particularly tailored for children with hydrocephalus who appear to have the more deficient skills.
\end{abstract}

Keywords Spinabifida $\cdot$ Myelomeningocele $\cdot$ Hydrocephalus $\cdot$ General Intelligence QI $\cdot$ Neuropsychological profiles $\cdot$ Executive function assessment

\section{Introduction}

Spina bifida (myelomeningocele) is one of the most complex birth defects compatible with life, with an estimated prevalence between 3.06 and 3.13 cases per 10,000 live births [1].

C. Rendeli

claudia.rendeli@policlinicogemelli.it

1 Dipartimento Scienze della salute della donna, del bambino e di sanità pubblica - Unità Operativa Spina Bifida e Uropatie Malformative, Fondazione Policlinico Universitario A. Gemelli IRCCS, Rome, Italy

2 Direzione Scientifica - Fondazione Policlinico Universitario A. Gemelli IRCCS, Rome, Italy

3 Scienze dell'invecchiamento, neurologiche, ortopediche e della testa-collo, Rome, Italy
Myelomeningocele is the most common form of "spina bifida," but other forms of open and closed lesions exist, including lipomyelomeningocele, diastematomyelia, diplomyelia, myelocystocele, myeloschisis, and fatty filum [2]. Spina bifida is the most common central nervous system birth defect encountered by the pediatric neurosurgeon. It is defined by characteristic development abnormalities of the vertebrae and spinal cord and associated changes in the cerebrum, brainstem, and peripheral nerves. As a result of denervation, muscle imbalance ensues and can result in abnormalities at the hip, knee, and foot. Anesthesia of various portions of the skin can lead to pressure sores, particularly later in life. Anorectal neuropathy may cause a variety of defecatory dysfunctions. Urologic abnormalities are also common. These multisystem abnormalities associated with spina bifida contribute to its widely accepted identity as the most complex development defect compatible with long life [3]. With 
advances in medical care, more individuals with spina bifida are surviving to adulthood $[4,5]$. A total of 43 Italian children, aged between 6 and 16 years, diagnosed with spina bifida, myelomeningocele, and shunted hydrocephalus, followed in Spina Bifida Centre of Policlinico Universitario A. GemelliRoma, have been described clinically and completed a neuropsychological battery in order to evaluate their profile.

\section{Materials and methods}

A total of 43 Italian children and young adults diagnosed with spina bifida completed a neuropsychological assessment battery in order to have their profiles evaluated. Enrolled children underwent cognitive assessment by means of the Weschler WISC-IV cognitive test and assessment of the attention sustained through the LEITER test. In addition, parents were asked, in order to obtain a personality and behavior profile of the children, to fill in a "CBCL 6-18 years" questionnaire and to fill in a Barthel Index questionnaire. Informed consent was signed by the parents. The protocol for this study was approved by the Ethics Committee of the Fondazione Policlinico A. Gemelli IRCCS, ID number: 3218 (2020).

\section{Neuropsychological tests}

The Wechsler Intelligence Scale for Children-IV (WISC-IV) is the clinical tool par excellence administered individually, to assess the cognitive abilities of children aged between 6 years and 0 months and 16 years and 11 months. The structure of the scale has been updated to reflect the theory and practice of assessing children, which implies increasing attention to working memory and processing speed. With the WISC-IV, 5 composite scores can be calculated: a total intellectual quotient to represent the complex cognitive abilities of the child, and 4 additional scores: the Verbal Comprehension Index, the Perceptual Reasoning Index, the Working Memory Index, and the Processing Speed Index. The Test Leiter-R International Performance Scale - Revised "attention sustained" is defined as the ability to maintain attention for a long time on "boring" tasks. The Child Behavior Checklist (CBCL, Achenbach, 6-18 years) version for parents is structured around 8 syndromic scales: anxiety/depression, withdrawal/depression, somatic complaints, social problems, thinking problems, attention problems, transgression behavior rules, and aggressive behavior, which are grouped into two other general dimensions, internalization and outsourcing.

\section{Statistical analysis}

The sample has been described in its clinical and demographic characteristics using descriptive statistics techniques. Qualitative variables have been presented with absolute frequencies and percentages $(n, \%)$, and quantitative variables have been summarized with mean ( \pm standard deviation). Normality of data has been verified using KolmogorovSmirnov test. Patients have been classified according to gait (autonomous, wheelchair, and tutor) and according to a dichotomized version of the diagnosis: lipoma, lipomyelomeningocele, and myelomeningocele (LMMC and MMC). Comparisons of categorical variables have been performed with Chi-square test (or Fisher's exact test), and comparisons of continuous variables have been performed with Mann-Whitney $U$ test and Kruskal-Wallis test. A $p$ value $<0.05$ has been considered statistically significant.

\section{Results}

A total of 43 patients, 22 females $(51.2 \%)$ and 21 males (48.8\%), have been enrolled in the study. Mean age of the whole sample was 10.72 years $( \pm 3.1)$ with a minimum age of 6 years and a maximum age of 16 . As shown in Table 1, the majority of our patients completed primary school (21, $48.8 \%)$ and received a lipoma diagnosis $(22,51.2 \%)$ or a myelomeningocele (MMC) $(20,46.5 \%)$ or a lipomyelomeningocele (LMMC) diagnosis $(1,2.32 \%)$.

Table 1 Clinical and demographic characteristics of the whole sample $(n=43)$

\begin{tabular}{|c|c|c|}
\hline & Sample & Percentage \\
\hline \multicolumn{3}{|l|}{ Gender } \\
\hline $\mathrm{F}$ & 22 & 51.2 \\
\hline $\mathrm{M}$ & 21 & 48.8 \\
\hline \multicolumn{3}{|l|}{ Education } \\
\hline Primary & 21 & 48.8 \\
\hline Lower secondary & 14 & 32.6 \\
\hline Upper secondary & 8 & 18.6 \\
\hline \multicolumn{3}{|l|}{ Diagnosis } \\
\hline LIPOMA & 22 & 51.2 \\
\hline MMC+LMMC & 21 & 48.8 \\
\hline \multicolumn{3}{|l|}{ Hydrocephalus } \\
\hline No & 27 & 62.8 \\
\hline Yes & 16 & 37.2 \\
\hline \multicolumn{3}{|l|}{ VP shunt } \\
\hline No & 29 & 67.4 \\
\hline Yes & 14 & 32.6 \\
\hline \multicolumn{3}{|l|}{ Gait } \\
\hline Autonomous & 26 & 60.5 \\
\hline Tutor & 9 & 20.9 \\
\hline \multirow[t]{2}{*}{ Wheelchair } & 8 & 18.6 \\
\hline & Mean & Std. dev. \\
\hline Age & 10.72 & 3.065 \\
\hline Barthel Index & 14.91 & 5.079 \\
\hline
\end{tabular}


Hydrocephalus is present in 16 patients (37.2\%) and all of them have been diagnosed as myelomeningocele. Ventriculoperitoneal shunt is present in 14 patients (32.6\%). Most of the patients were in a condition of autonomous gait $(26,60.5 \%)$. Barthel Index mean value was $14.91( \pm 5.1)$. Table 2 shows that WISC mean value was $88.83( \pm 19.1)$ and, in more detail, the Verbal Comprehension Index mean value was $96.58( \pm 16.7)$, the Working Memory mean value was $83.56( \pm 18.6)$, and the Processing Speed Index mean value was $90.93( \pm 17.6)$. The distributions of categorical variables by gait highlighted a statistically significant difference in Barthel Index $(p=0.001)$ where patients with wheelchair presented the lowest Barthel Index mean value $(8.3 \pm 4.6)$ and the highest was presented by autonomous patients $(17.1 \pm$ 3.1). Also, the Processing Speed Index of the WISC-IV QI scale was statistically significant $(p=0.027)$, with the highest value presented by autonomous patients $(95.8 \pm 12.8)$ and the lowest by patients using a wheelchair $(75.5 \pm 19)$. See Table 3 for details. Table 4 presents the comparisons of patients according to diagnosis. WISC-IV QI mean value is $98( \pm 15.7)$ for lipoma patients and $78.7( \pm 17.6)$ for LMMC and MMC patients $(p=0.001)$. In more detail, Perceptual Reasoning $(p<$ $0.0005)$, Working Memory $(p=0.01)$, and Processing Speed index $(p=0.001)$ highlighted a significant difference between the groups. The attention sustained subscale of the LEITER presented a mean of $6.9( \pm 3.1)$ for lipoma patients and a men value of $4.6( \pm 3.1)$ for LMMC and MMC patients $(p=0.024)$. Table 5 reports a comparison between patients with and without hydrocephalus about cognition and autonomy. As shown, patients with hydrocephalus had worse cognition score regarding IQ $(p<0.001)$ and autonomy (Barthel Index) $(p<$ $0.001)$.

Bivariate analyses have shown that within those who are autonomous, there are no significant differences between lipoma and MMC patients in terms of neuropsychological test results, similarly among wheelchair users and among tutor users.

Table 2 Clinical characteristics of the whole sample $(n=43)$

\begin{tabular}{lcc}
\hline & Mean & Std. dev. \\
\hline WISC-IV QI & 88.83 & 19.088 \\
Verbal Comprehension Index & 96.58 & 16.783 \\
Perceptual Reasoning & 88.37 & 20.121 \\
Working Memory & 83.56 & 18.593 \\
Processing Speed Index & 90.93 & 17.667 \\
CBCL & & \\
Internalizing problems & 58.65 & 8.144 \\
Externalizing problems & 49.81 & 7.346 \\
Total problems & 50.23 & 8.106 \\
LEITER & & 3.263 \\
Attention sustained & 5.83 & \\
\hline
\end{tabular}

There is no statistically significant difference, for neuropsychological tests result, comparing patients without hydrocephalus and with different gait abilities. However, within patients with hydrocephalus, some tests gave significantly different results between gait ability groups: The Barthel Index shows significantly different results between "autonomous vs. wheelchair" ( $p=0.035)$ and "tutor vs. wheelchair" ( $p=$ 0.017). Perceptual reasoning presented significantly different results between "autonomous vs. wheelchair" $(p=0.042)$ and "tutor vs. wheelchair" $(p=0.013)$. Processing speed was significantly different between "autonomous vs. wheelchair" $(p$ $=0.033)$ and "tutor vs. wheelchair" $(p=0.031)$. LEITER attention sustained presented significantly different results between "autonomous vs. wheelchair" $(p=0.024)$ and "tutor vs. wheelchair" $(p=0.049)$. See Table 6 for details.

\section{Discussion}

Spina bifida malformation (SBM) is a neural tube defect originating in the first 30 days of gestation and associated with failure of the caudal end of the neural tube to close. It is a heterogeneous congenital disorder with complex physical and neuropsychological symptomatology. It is associated with developmental anomalies of both the spine and the central nervous system. Different forms of spina bifida can be distinguished from mild to severe. The most common form is myelomeningocele (MMC). Most patients with myelomeningocele present associated hydrocephalus. Hydrocephalus in SBM results from the Chiari II cerebellum and hindbrain malformations, which obstructs the flow of the cerebrospinal fluid (CSF) at the level of the third and fourth ventricles. Surgical management with ventriculoperitoneal shunt implantation (VPS) is a widely used, effective treatment for hydrocephalus. However, consistent follow-up is necessary after VPS, taking into consideration several shunt-related complications including obstruction, fracture, and infection. Also, the endoscopic third ventriculostomy (ETV) procedure is well recognized as an effective alternative to cerebrospinal fluid (CSF) shunt placement and has been the ideal treatment of choice for obstructive hydrocephalus in recent years. As for the neurocognitive aspects, children with hydrocephalus and SBM are more impaired in spatial, math, memory, and concept formation domains, compared to their relative strengths in vocabulary and word reading domains [6, 7]. Within content domains, cognitive strengths involve the learned association and categorization of stimulus information, such as word recognition, vocabulary, and priming abilities. Areas of weakness involve abstract assembly and construction of information, such as coordinating visual perception, mathematical computation, reading and language comprehension, and concept formation tasks [6-8]. The purpose of this study was to observe the psychological and neuropsychological condition of children 
Table 3 Distributions of some continuous variables by gait

\begin{tabular}{|c|c|c|c|c|c|c|c|c|c|c|}
\hline \multirow[t]{2}{*}{ Gait } & \multicolumn{3}{|c|}{ Autonomous } & \multicolumn{3}{|c|}{ Wheelchair } & \multicolumn{3}{|c|}{ Tutor } & \multirow{2}{*}{$\begin{array}{l}p \text { - } \\
\text { value }\end{array}$} \\
\hline & $n$ & Mean & Std. dev. & $n$ & Mean & Std. dev. & $n$ & Mean & Std. dev. & \\
\hline Age & 26 & 10.4 & 3.0 & 8 & 12.8 & 2.6 & 9 & 9.8 & 3.2 & 0.087 \\
\hline Barthel Index autonomia & 26 & 17.1 & 3.1 & 8 & 8.3 & 4.6 & 9 & 14.6 & 5.1 & 0.001 \\
\hline WISC-IV QI & 26 & 92.2 & 14.1 & 7 & 75.9 & 22.9 & 9 & 89.3 & 25.8 & 0.251 \\
\hline Verbal Comprehension Index & 26 & 98.5 & 11.9 & 8 & 89.0 & 22.7 & 9 & 97.7 & 22.6 & 0.719 \\
\hline Perceptual Reasoning & 26 & 93.0 & 15.5 & 8 & 70.6 & 25.1 & 9 & 90.8 & 20.8 & 0.054 \\
\hline Working Memory & 26 & 87.4 & 15.7 & 8 & 73.0 & 17.1 & 9 & 81.8 & 25.0 & 0.176 \\
\hline Processing Speed Index & 26 & 95.8 & 12.8 & 8 & 75.5 & 19.0 & 9 & 90.6 & 22.3 & 0.027 \\
\hline \multicolumn{11}{|l|}{ CBCL } \\
\hline Internalizing problems & 26 & 58.8 & 8.6 & 8 & 57.4 & 6.0 & 9 & 59.2 & 9.1 & 0.782 \\
\hline Externalizing problems & 26 & 50.2 & 8.4 & 8 & 49.3 & 5.5 & 9 & 49.3 & 5.9 & 0.948 \\
\hline Total problems & 26 & 50.3 & 9.0 & 8 & 48.9 & 4.8 & 9 & 51.3 & 8.2 & 0.865 \\
\hline \multicolumn{11}{|l|}{ LEITER } \\
\hline Attention sustained & 26 & 6.6 & 3.0 & 7 & 3.4 & 3.7 & 8 & 5.5 & 3.0 & 0.079 \\
\hline
\end{tabular}

affected by spina bifida, a condition with a great severity and clinical variety. The study showed that lipoma patients have a more adequate cognitive function development than MMC patients. Moreover, patients with autonomous ambulation (most of them are lipoma patients) presented a normal cognitive development unlike patients in need of a wheelchair or a tutor. Patients that need a wheelchair are the most affected both clinically and cognitively. Considering the components of cognitive processes (WISC-IV), such as Perceptual Reasoning, Working Memory, and Processing Speed Index, the study proved that these skills are preserved in lipoma patients rather than in MMC patients who have difficulties in these skills due to the severity of their pathology. Particularly, the Processing Speed ability is linked with concentration and attention, cognitive flexibility, visual ability, and organizing an autonomous task fast. These results can be useful in planning dedicated therapeutic protocols such as suitable rehabilitation treatments, speech therapy, psychomotor skills, and cognitive enhancement. Cognitive ability scores, such as Working Memory (WISC-IV) and Sustained Attention (LEITER), are in both lipoma and LMMC and MMC patients below normal values and/or slightly below normal values $[9,10]$.

From a behavioral point of view, the analysis of the questionnaires filled by the parents highlights that no clinical or pathological scores are present in the three scales investigated by the CBCL tool. Results highlight that children, even if they do not have clinical scores, tend to use internalizing defense mechanisms, showing an attitude characterized by greater

Table 4 Distributions of some continuous variables by diagnosis

\begin{tabular}{|c|c|c|c|c|c|c|c|}
\hline & \multicolumn{3}{|c|}{ Lipoma } & \multicolumn{3}{|c|}{ LMMC and MMC } & \multirow[t]{2}{*}{$p$-value } \\
\hline & $n$ & Mean & Std. dev. & $n$ & Mean & Std. dev. & \\
\hline Age & 22.0 & 10.4 & 2.8 & 21.0 & 11.1 & 3.4 & 0.501 \\
\hline Barthel Index autonomia & 22.0 & 16.4 & 4.0 & 21.0 & 13.3 & 5.7 & 0.072 \\
\hline WISC-IV QI & 22.0 & 98.0 & 15.7 & 20.0 & 78.7 & 17.6 & 0.001 \\
\hline Verbal Comprehension Index & 22.0 & 102.5 & 14.0 & 21.0 & 90.4 & 17.6 & 0.063 \\
\hline Perceptual Reasoning & 22.0 & 99.2 & 16.9 & 21.0 & 77.0 & 16.9 & $<0.0005$ \\
\hline Working Memory & 22.0 & 90.7 & 16.0 & 21.0 & 76.0 & 18.5 & 0.010 \\
\hline Processing Speed Index & 22.0 & 99.8 & 13.3 & 21.0 & 81.6 & 17.1 & 0.001 \\
\hline \multicolumn{8}{|l|}{ CBCL } \\
\hline Internalizing problems & 22.0 & 58.7 & 8.8 & 21.0 & 58.6 & 7.6 & 0.903 \\
\hline Externalizing problems & 22.0 & 50.5 & 8.5 & 21.0 & 49.0 & 6.1 & 0.557 \\
\hline Total problems & 22.0 & 50.6 & 8.8 & 21.0 & 49.9 & 7.5 & 0.826 \\
\hline LEITER attention sustained & 22.0 & 6.9 & 3.1 & 19.0 & 4.6 & 3.1 & 0.024 \\
\hline
\end{tabular}


Table 5 Distributions of some continuous variables by presence of hydrocephalus

\begin{tabular}{|c|c|c|c|c|c|c|c|}
\hline & \multicolumn{3}{|c|}{ Without hydrocephalus } & \multicolumn{3}{|c|}{ With hydrocephalus } & \multirow[t]{2}{*}{$p$-value } \\
\hline & $n$ & Mean & Std. dev. & $n$ & Mean & Std. dev. & \\
\hline Age & 26 & 10.3 & 2.8 & 16 & 11.7 & 3.3 & 0.167 \\
\hline Barthel Index autonomia & 26 & 16.6 & 3.7 & 16 & 12.0 & 5.9 & 0.010 \\
\hline WISC-IV QI & 26 & 96.7 & 15.2 & 15 & 75.7 & 18.7 & 0.001 \\
\hline Verbal Comprehension Index & 26 & 102.8 & 13.3 & 16 & 86.6 & 17.9 & 0.008 \\
\hline Perceptual Reasoning & 26 & 96.8 & 16.6 & 16 & 75.1 & 18.9 & 0.001 \\
\hline Working Memory & 26 & 90.7 & 15.4 & 16 & 72.5 & 18.7 & 0.003 \\
\hline Processing Speed Index & 26 & 97.7 & 13.6 & 16 & 80.2 & 19.1 & 0.003 \\
\hline \multicolumn{8}{|l|}{ CBCL } \\
\hline Internalizing problems & 26 & 58.0 & 9.0 & 16 & 59.6 & 7.1 & 0.622 \\
\hline Externalizing problems & 26 & 50.0 & 7.9 & 16 & 49.0 & 6.3 & 0.845 \\
\hline Total problems & 26 & 50.1 & 8.5 & 16 & 50.3 & 7.9 & 0.917 \\
\hline LEITER attention sustained & 26 & 6.6 & 3.1 & 14 & 4.2 & 3.2 & 0.023 \\
\hline
\end{tabular}

emotional closure and social withdrawal rather than externalizing attitude (outbursts or aggression).

Study's results highlighted that children with spina bifida, checked from birth constantly each 6-12 months, have a normal cognitive functioning characterized by difficulties in working memory, which is slightly lower than the normal value, but with a strength in verbal understanding. These findings can guide a better assessment of patients' needs in order to develop prevention protocols (suitable rehabilitation treatments such as speech therapy, psychomotor skills, cognitive enhancement) particularly tailored for children with hydrocephalus who appear to have the more deficient skills. About the presence of hydrocephalus, the authors observed that these patients have worse score both for cognitive intelligent quotient analyzed with the WISCH scale and for autonomy with the Barthel Index. These results can be explained because patients with hydrocephalus have severe clinical manifestations of spina bifida and also because hydrocephalus causes a worse impact on quality of life and cognitive performances.

\section{Conclusions}

This study underlined that spina bifida patients should also be evaluated for their neuropsychological deficits in order to organize specific rehabilitation programs.

Table 6 Distributions of neuropsychological results by gait abilities in the hydrocephalus group

\begin{tabular}{|c|c|c|c|c|c|c|c|c|c|c|}
\hline & \multicolumn{10}{|c|}{ With hydrocephalus } \\
\hline & \multicolumn{3}{|c|}{ Autonomous } & \multicolumn{3}{|c|}{ Wheelchair } & \multicolumn{3}{|c|}{ Tutor } & \multirow{2}{*}{$\begin{array}{l}p \text { - } \\
\text { value }\end{array}$} \\
\hline & $n$ & Mean & Std. dev. & $n$ & Mean & Std. dev. & $n$ & Mean & Std. dev. & \\
\hline Age & 3 & 13.7 & 2.1 & 6 & 12.5 & 3.0 & 7 & 10.1 & 3.6 & 0.3 \\
\hline Barthel Index autonomia & 3 & 15.7 & 2.1 & 6 & 7.0 & 3.5 & 7 & 14.7 & 5.9 & 0.0 \\
\hline WISC-IV QI & 3 & 79.7 & 8.5 & 5 & 64.4 & 14.6 & 7 & 82.1 & 22.2 & 0.2 \\
\hline Verbal Comprehension Index & 3 & 86.7 & 8.3 & 6 & 81.7 & 21.5 & 7 & 90.7 & 18.6 & 0.6 \\
\hline Perceptual Reasoning & 3 & 84.0 & 9.5 & 6 & 58.3 & 12.1 & 7 & 85.7 & 17.0 & 0.0 \\
\hline Working Memory & 3 & 75.0 & 11.4 & 6 & 68.5 & 16.4 & 7 & 74.9 & 24.1 & 0.8 \\
\hline Processing Speed Index & 3 & 92.0 & 9.2 & 6 & 66.3 & 10.0 & 7 & 87.0 & 22.1 & 0.0 \\
\hline \multicolumn{11}{|l|}{ CBCL } \\
\hline Internalizing problems & 3 & 59.7 & 11.0 & 6 & 57.7 & 6.9 & 7 & 61.1 & 6.3 & 0.6 \\
\hline Externalizing problems & 3 & 44.3 & 9.6 & 6 & 50.0 & 4.5 & 7 & 50.1 & 6.2 & 0.6 \\
\hline Total problems & 3 & 47.0 & 14.4 & 6 & 48.7 & 4.9 & 7 & 53.1 & 7.1 & 0.6 \\
\hline LEITER attention sustained & 3 & 7.0 & 3.5 & 5 & 1.6 & 1.3 & 6 & 5.0 & 2.8 & 0.0 \\
\hline
\end{tabular}


Availability of data and material (data transparency) The datasets used and/or analyzed during the current study are available from the corresponding author on reasonable request.

Funding Open access funding provided by Università Cattolica del Sacro Cuore within the CRUI-CARE Agreement.

\section{Declarations}

Ethics approval (include appropriate approvals or waivers) ID No. 3218 (2020).

\section{Consent to participate (include appropriate statements) Yes.}

Consent for publication (include appropriate statements) Yes.

Conflict of interest The authors have no competing interests, or other interests that might be perceived to influence the interpretation of the article.

Open Access This article is licensed under a Creative Commons Attribution 4.0 International License, which permits use, sharing, adaptation, distribution and reproduction in any medium or format, as long as you give appropriate credit to the original author(s) and the source, provide a link to the Creative Commons licence, and indicate if changes were made. The images or other third party material in this article are included in the article's Creative Commons licence, unless indicated otherwise in a credit line to the material. If material is not included in the article's Creative Commons licence and your intended use is not permitted by statutory regulation or exceeds the permitted use, you will need to obtain permission directly from the copyright holder. To view a copy of this licence, visit http://creativecommons.org/licenses/by/4.0/.

\section{References}

1. Burro F, Cama A, Lertora V, Veneselli E, Rossetti S, Pezzuti L (2018) Intellectual efficiency in children and adolescents with spina bifida myelomeningocele and shunted hydrocephalus. Dev Neuropsychol 43(3):198-206. https://doi.org/10.1080/87565641. 2018.1439035 Epub 2018 Feb 16

2. Wasserman RM, Holmbeck GN (2016) Profiles of neuropsychological functioning in children and adolescents with spina bifida: associations with biopsychosocial predictors and functional outcomes. J Int Neuropsychol Soc. 22(8):804-815. https://doi.org/10. 1017/S1355617716000680 Epub 2016 Aug 30

3. Vinck A, Nijhuis-van der Sanden MW, Roeleveld NJ, Mullaart RA, Rotteveel JJ, Maassen BA (2010) Motor profile and cognitive functioning in children with spina bifida. Eur J Paediatr Neurol. 14(1): 86-92. https://doi.org/10.1016/j.ejpn.2009.01.003 Epub 2009 Feb 23

4. Iddon JL, Morgan DJ, Loveday C, Sahakian BJ, Pickard JD (2004) Neuropsychological profile of young adults with spina bifida with or without hydrocephalus. J Neurol Neurosurg Psychiatry 75(8): 1112-1118. https://doi.org/10.1136/jnnp.2003.029058

5. Iddon JL, Morgan DJ, Ahmed R, Loveday C, Sahakian BJ, Pickard JD (2003) Memory and learning in young adults with hydrocephalus and spina bifida: specific cognitive profiles. Eur J Pediatr Surg. 13(Suppl 1):S32-S35

6. Barnes MA, Dennis M (1998) Discourse after early-onset hydrocephalus: core deficits in children of average intelligence. Brain Lang 61(3):309-334. https://doi.org/10.1006/brln.1998.1843

7. Dennis et al (2006) Motor learning in children with spina bifida: intact learning and performance on a ballistic task. J Int Neuropsychol Soc 12(5):598-608. https://doi.org/10.1017/ S1355617706060772

8. Dennis M, Nelson R, Jewell D, Fletcher JM (2010) Prospective memory in adults with spina bifida. Childs Nerv Syst 26(12): 1749-1755. https://doi.org/10.1007/s00381-010-1140-z

9. Pollenus J, Lagae L, Aertsen M, Jansen K (2020) The impact of cerebral anomalies on cognitive outcome in patients with spina bifida: a systematic review. Eur J Paediatr Neurol 28:16-28

10. Kritikos TK, Smith K, Holmbeck GN (2020) Mental health guidelines for the care of people with spina bifida. J Pediatr Rehabil Med. 13(4):525-534. https://doi.org/10.3233/PRM-200719

Publisher's note Springer Nature remains neutral with regard to jurisdictional claims in published maps and institutional affiliations. 